Rickettsia massiliae $(\mathrm{n}=1)$, Babesia canis canis $(\mathrm{n}=3)$, Babesia gibsoni $(\mathrm{n}=2)$ and similar sequences of hemotropic Mycoplasma spp. $(\mathrm{n}=2)$, in blood; Leishmania infantum $(\mathrm{n}=1)$, Ehrlichia canis $(n=1)$, Babesia canis canis $(n=1)$ and Babesia gibsoni $(\mathrm{n}=1)$, in spleen. Two control dogs (3.3\%) showed positivity to Babesia gibsoni $(\mathrm{n}=1)$ and Hepatozoon canis $(n=1)$. The $58.3 \%$ of dogs infected presented benign lesions (7/12), 3 with IBD; the remaining $41.7 \%$ were diagnosed with malignancies $(5 / 12)$.

Infection resulted a predisposing factor for splenic diseases ( $p=0.004, O R=7.56)$. Relationship between the type of splenic lesion and positivity to VBP was not significant $(p=0.94)$, although association between Babesia canis canis and hemangiosarcoma was found $(p=0.004)$. IBD was related to detection of VBP $(p=0.02)$ and the presence of splenic lesions $(p=0.025)$ but not to the type of splenic disease $(p=0.123)$.

Main findings suggest that positivity to VBP could be related to splenic diseases. We hypothesize that the relevant immunological role of the spleen could predispose to alterations of this organ in the presence of VBP. Furthermore, Bartonella spp. has been proposed to be involved in the pathogenesis of vasoproliferative tumors. Interestingly, all dogs with Babesia canis canis presented hemangiosarcoma in the present survey. The real relationship between IBD, splenic diseases and positivity to VBP could not be established. Coincidental with previous publications, results support that diagnosis of VBP could be improved by analysis of samples from different tissues. The number of dogs included here warrants further investigation.

\section{Antibacterial dispensing behaviour in a large sentinel network of dog and cat practices in the UK}

\section{David Singleton, Fernando Sanchez- Vizcaino Buendia, P-J Noble, Philip Jones, Gina Pinchbeck, Susan Dawson, Nicola Williams, Alan Radford}

University of Liverpool, Neston, UK

Antimicrobial resistance (AMR) has been described as one of the greatest current challenges to the future of both veterinary and human health, with antibacterial usage a key driver in AMR development. However, information on how antibacterials are dispensed, particularly in companion animals, has to date been limited, restricting the ability of the veterinary profession to respond to the challenge of AMR in an informed manner. The Small Animal Veterinary Surveillance Network (SAVSNET) is a collaborative project between the University of Liverpool and the BSAVA, which collects large volumes of electronic health records from UK veterinary practices and diagnostic laboratories to improve companion animal-disease surveillance at local, regional and national scales.

In addition to health data and the owner's full postcode gathered in real-time at each consultation, SAVSNET captures the 'items dispensed'. A regular expressions approach was utilised to produce a list of word roots $(n=366)$ to identify dispensed antibacterial products. Antibacterial dispensing consultations were then identified with data collected over an 18-month period (17/03/2014 to 24/09/2015) from 307 veterinary sites across the UK, consisting of 391,132 canine (175,789 dogs) and 154,222 feline consultations (87,247 cats). Proportions and confidence intervals (95\%) were calculated using robust standard errors to allow for the clustering within individual veterinary sites.

For dogs and cats, 83,697 (21.4\%) and 31,297 (20.3\%) of all consultations respectively involved at least one antibacterial-containing product being dispensed. In dogs, 83.6\% (81.9-85.3); 11.3\% (9.7-12.9) and 5.1\% (4.5-5.7) of dispensed antibacterial products were veterinary-licenced; human-licenced or generic dual-licenced drugs, respectively. This compared with $90.4 \%$ (89.6-91.2); 4.9\% (4.4-5.4) and $4.7 \%(4.1-5.3)$ in cats. The most commonly dispensed antibacterial in dogs was clavulanic-acid-potentiated-amoxicillin (28.2\% (26.7-29.7) of antibacterial products dispensed), consistent with previous studies. For cats, the most commonly dispensed antibacterial was cefovecin (34.8\% (31.438.2)), in contrast to an earlier study where amoxicillin was most commonly dispensed. Fluoroquinolones comprised $4.7 \%$ (3.8-5.6) and 3.3\% (1.6-5.0) of dispensed antibacterials in dogs and cats, respectively. During the study period, no vancomycin or teicoplanin (glycopeptides); meropenem or imipenem (carbapenems) containing-products were dispensed in any animal species.

The methodology describes a near real-time antibacterial dispensing surveillance system that will allow SAVSNET to describe geographical and temporal dispensing trends, and dispensing based on syndromic information. As demonstrated, the system will also allow active monitoring for veterinary usage of key antibacterials recommended as 'reserved' for human health. 